\title{
MODELO AHP PARA SELECCIONAR PROVEEDORES DE CLOUD COMPUTING
}

\section{AHP MODEL TO SELECT SUPPLIERS CLOUD COMPUTING}

\author{
Gloria Teresita Huamaní Huamaní ${ }^{1}$ \\ RESUMEN
}

En este documento se describe la aplicación del Proceso Analítico Jerárquico (PAJ), y luego se identifica los servicios de los proveedores de computación en la nube. La primera actividad en la toma de decisiones multicriterio es establecer los factores que son importantes para esa decisión. Así se ha determinado como criterios en la selección de proveedores de cloud computing: escalabilidad, accesibilidad, rendimiento, y seguridad. El modelo SEPROCCAHP v01, ha sido aplicado por tres grupos de alumnos del curso de logística empresarial. Dos grupos de alumnos han seleccionado Google, y un tercer grupo a Microsoft. Los resultados difieren, porque depende de los juicios de valor emitidos en la evaluación de pares de criterios y las alternativas a seleccionar.

Palabras clave.- Seleccionar proveedores, Proceso analítico jerárquico, Computación en la nube.

\begin{abstract}
In this paper the application of the Analytic Hierarchy Process (AHP) is described, and then the service providers cloud computing is identified. The first activity in multicriteria decisionmaking is to establish the factors that are important to that decision. This has been determined as criteria in selecting cloud providers: accessibility scalability, performance, security. The SEPROCCAHP v01 model has been applied for three groups of students in the course of business logistics. Two groups of students have selected Google, and a third group at Microsoft. The results differ because it depends on value judgments issued in the peer evaluation criteria and alternatives to select.
\end{abstract}

Key words- Select suppliers, Analytic hierarchy process, Cloud computing.

\section{INTRODUCCIÓN}

El objetivo de este trabajo es proponer un modelo AHP en la selección de proveedores de Cloud Computing, y observar a tres grupos de investigación en la aplicación del modelo.

Para proponer el modelo se ha descrito, por un lado el Proceso Analítico Jerárquico (PAJ)/ Analityc Hierarchy Process (AHP) y por otra parte se hace una revisión teórica acerca de niveles de servicio de Cloud Computing, de este modo se ha establecido cuatro criterios entre varios que existen.
La determinación de criterios y selección de proveedores de Cloud Computing, rebasa el ámbito del curso de logística empresarial, la investigación sobre el tema requiere tiempo, recursos y equipo multidisciplinario.

La aplicación del modelo jerárquico "Selección de proveedores de cloud computing mediante AHP" (SEPROCCAHP) v01, ha sido realizada por tres grupos de estudio del curso de logística empresarial, curso de octavo ciclo. Este documento contiene: selección de proveedores y planeación del suministro, proceso analítico jerárquico, proveedores de Cloud Computing,

\footnotetext{
${ }^{1}$ Dr. Docente principal de logística empresarial en la Facultad de Ingeniería Industrial y de Sistemas de la Universidad Nacional de Ingeniería.
} 
aplicación de AHP en selección de proveedores de Cloud Computing, y análisis comparativo de tres grupos de investigación.

\section{SELECCIÓN DE PROVEEDORES Y PLANEACIÓN DEL SUMINISTRO}

En la gestión de cadenas de suministros (GCS)/ supply chain management (SCM), hay tres flujos, tales como: flujo de materiales, flujo de recursos financieros y flujo de la información.

Toma una importancia singular la interrelación entre la compañía y los proveedores, asimismo entre la compañía y los clientes, en las que se realiza procesos que involucran actividades diversas (ver Figura 1).

\section{PROCESOS DE LA CADENA DE SUMINISTROS}

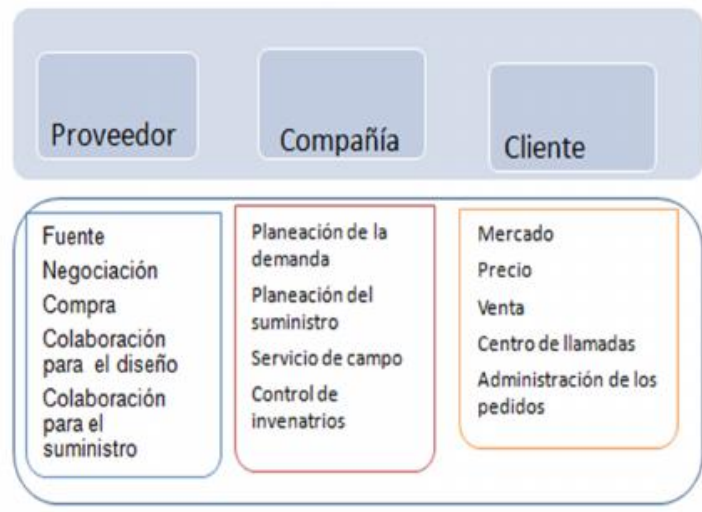

Fig. 1 Planeación de GCS/SCM, adaptado de Chopra [1].

Una óptima relación entre el proveedor y la compañía se vislumbra en la colaboración para el diseño y para el suministro; una actividad imprescindible como la selección de proveedores es parte del proceso de la planeación del suministro. Con la finalidad de eliminar el grado de subjetividad en la evaluación y en el proceso de Toma de decisiones se aplican técnicas, métodos y modelos que coadyuvan a que el proceso sea transparente. En este documento se muestra la aplicación del Proceso Analítico Jerárquico (PAJ) en la selección de proveedores (sección 4).

\section{PROCESO ANALÍTICO JERÁRQUICO (PAJ)/ ANALITYC HIERARCHY PROCESS (AHP)}

El proceso de análisis jerárquico (PAJ/AHP), es una técnica desarrollada por Saaty en 1977, y propuesto de manera integrada en su libro en 1980. Saaty ha publicado diversos modelos jerárquicos aplicando a problemas complejos tanto políticos, económicos, sociales, administrativos, etc. Saatty, en 1990 [2] responde a la pregunta ¿Cómo estructurar un problema de decisión? "Tal vez la tarea más creativa en la toma de decisiones es elegir los factores que son importantes para esa decisión. En el Proceso Analítico Jerárquico se organiza estos factores, una vez seleccionados, en una estructura jerárquica descendiendo de la meta global, a criterios, subcriterios y alternativas en niveles sucesivos. Cuando se construye jerarquías se debe incluir suficiente detalle relevante para: representar el problema lo más detallado posible sin perder la sensibilidad de ver los cambios de los elementos; considerar el medio ambiente que rodea el problema; identificar los atributos que contribuyen a la solución; e identificar a los participantes asociados con la solución del problema"

\section{Descripción de pasos AHP}

Paso 1: Desarrollo de la estructura jerárquica.- La construcción de un modelo jerárquico permite al decisor estructurar el problema en forma visual, con la técnica se busca formalizar la comprensión intuitiva de un problema multicriterio complejo.

En Figura 2, se muestra los niveles de la jerarquía: Meta Global, Criterios y Alternativas.

Paso 2: Representación de los juicios de valor.AHP permite que las personas utilicen su propia escala psicométrica para realizar las comparaciones entre dos opciones (criterios). Los decisores emiten juicios en términos de preferencia, de importancia o de probabilidad utilizando una escala numérica. Saaty propone una escala de 1 a 9, en Tabla 1, se muestra la escala verbal con su equivalente en números. 
Fig. 2 Modelo Jerárquico.

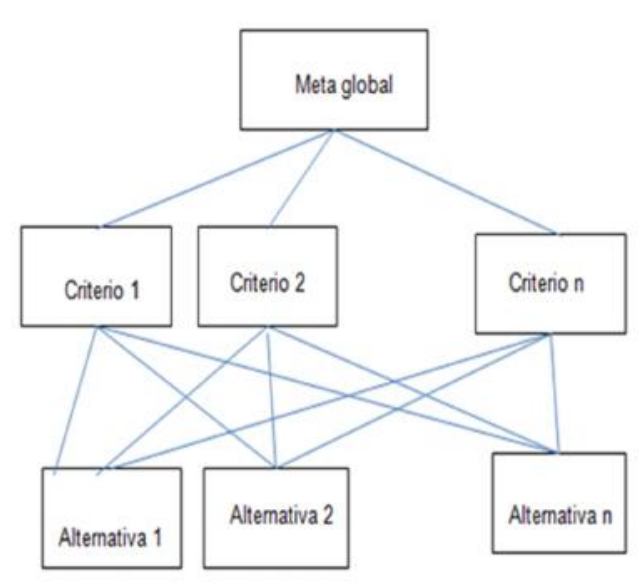

Tabla 1. Escala de juicios de valor.

\begin{tabular}{ll}
\hline $\begin{array}{l}\text { Escala verbal o } \\
\text { preferencia }\end{array}$ & $\begin{array}{l}\text { Evaluación } \\
\text { numérica }\end{array}$ \\
\hline $\begin{array}{l}\text { lgualmente preferido } \\
\text { Moderada preferido }\end{array}$ & 1 \\
Fuertemente preferido & 5 \\
$\begin{array}{l}\text { Muy fuertemente } \\
\text { preferido }\end{array}$ & 7 \\
$\begin{array}{l}\text { En extremo preferido } \\
\text { Valores intermedios }\end{array}$ & 9 \\
$\begin{array}{l}\text { Si a la actividad i se le ha } \\
\text { asignado uno de los }\end{array}$ & Recíprocos \\
números distintos a cero \\
mencionados, cuando se \\
compara con la j entonces \\
j tiene le recíproco \\
$\begin{array}{l}\text { cuando se le compara con } \\
\text { i } \\
\text { Coeficientes que surgen } \\
\text { de la escala }\end{array}$ \\
\hline
\end{tabular}

Fuente. Saaty.

Paso 3 construcción de las matrices de juicio de valor.- La jerarquía de criterios y alternativas es el objeto de comparación por pares para el AHP.
Paso 4: Cálculos a desarrollar.- Una vez construida las matrices se pasa a realizar los siguientes cálculos: cálculo de los vectores y cálculo de la consistencia. A la construcción de matrices de comparaciones de pares, se le aplica el operador vector propio para derivar los pesos de los criterios, y su correspondiente valor propio.

\section{Medida de la inconsistencia}

La medida propuesta por Saaty (1980) para evaluar la inconsistencia de una matriz pareada $\mathrm{A}$ $n * n=a(i j)$, llamada Razón de Consistencia de Saaty $(R C)$, viene dada por

$$
\begin{gathered}
R C=\frac{I C}{I R(n)} d o n d e \\
I C=\frac{\lambda \max (A)-1}{n-1}=\frac{1}{n(n-1)} \sum_{i \neq j}^{n}(e i j-1)
\end{gathered}
$$

siendo $\lambda \max$ el valor propio principal de la matriz $A$,

$I R(n)$ el índice de consistencia aleatorio para matrices de orden $n, \mathrm{y}$

$e i j=\operatorname{aij}\left(\frac{\omega \mathrm{j}}{\omega \mathrm{i}}\right)$ el error obtenido al estimar aij por medio de $\left(\frac{\omega \mathrm{j}}{\omega \mathrm{i}}\right)$

\section{PROVEEDORES DE CLOUD}

Cloud Computing tiene diversas definiciones y la oferta de servicios de es variada, depende del tipo de servicio. Para INTECO [3]. El Cloud Computing nace como una nueva alternativa o modelo de servicio tecnológico basado en la optimización de costes, eficiencia y flexibilidad para el acceso a los recursos tecnológicos por parte de las organizaciones, ciudadanos y entidades públicas.

Varios autores, entre ellos, Rodríguez, Pettorutti, et. al. [4] afirman que las Cloud suelen ser explotadas bajo un modelo de pago, acorde al uso, donde el proveedor garantiza las capacidades y posibilidades por medio de acuerdos de nivel de servicio.

Para Google, Cloud es un conjunto de aplicaciones y plataformas basadas en una infraestructura 
compartida, a la que se accede desde un navegador web y cuya modalidad de pago es por uso. [5].

\section{CLASIFICACION DE SOLUCIONES DE CLOUD COMPUTING}

Según INTECO [op. cit 3] en un modelo Cloud, las organizaciones pueden pasar de la gestión tecnológica tradicional (con cuantiosas inversiones en recursos, incluyendo hardware, software, centros de procesamiento de datos, redes, personal, seguridad, etc.), al nuevo modelo de gestión en la nube, donde se elimina la necesidad de grandes inversiones y costes fijos y se transforma a los proveedores en instrumentos que ponen al alcance de los usuarios, de forma flexible e instantánea, la capacidad de computación bajo demanda. Cloud Computing se clasifica de acuerdo al despliegue o de acuerdo al nivel de servicio.

\section{Clasificación de Cloud Computing por modelo de despliegue}

Existe Cloud pública, privada, e híbrida comunitaria

\section{Clasificación de Cloud por nivel de servicio}

Las arquitecturas Cloud se agrupan y clasifican por los servicios que prestan: Software como Servicio/ Software as a Service (SaaS), Plataformas como Servicios/ Platform as a Service (PaaS) e Infraestructura y Servicios/ Infrastructure as a Service (IaaS), (ver Figura 3).

\section{Software como Servicio}

Se considera a aquellas aplicaciones de software disponibles en Internet y que se las puede utilizar sin necesidad de ser localmente instaladas en una computadora.

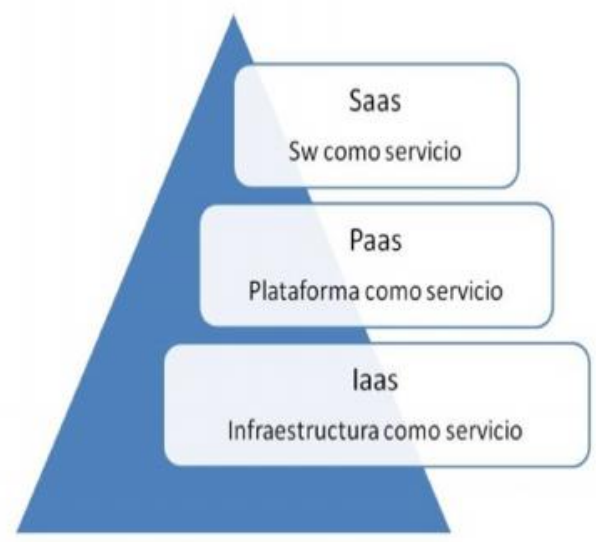

Fig. 3 Clasificación por servicio de Cloud Computing adaptado de Rodríguez [Op. cit].

\section{Plataforma como servicio}

El Cloud proporciona entornos configurados con todas las herramientas de software necesarias para el desarrollo y/o despliegue de aplicaciones personalizadas, sin que el usuario se preocupe en la instalación y administración de la infraestructura.

\section{Infraestructura como servicio}

Por medio de la tecnología de virtualización de recursos de hardware, Cloud Computing permite proporcionar recursos de infraestructura virtual (servidores, equipos, dispositivos de almacenamiento, dispositivos de red, entre otros.).

Los servicios de Cloud facilitan el soporte de TI a distancia, y ofrecen movilidad de la información, gracias a que se puede acceder a la misma desde cualquier parte, a cualquier hora y desde cualquier dispositivo, al transferir la información de un servidor interno a un servidor virtual. Un Cloud necesita satisfacer requerimientos de usuarios y los modelos de servicio tienen en cuenta las aplicaciones, la plataforma tecnológica, la infraestructura y virtualización, según Tiago [6].

Desde hace tiempo se utiliza ciertas formas de Cloud Computing, por ejemplo las Redes Sociales como Facebook/MySpace y cuentas de correo electrónico basadas en Web como gmail. 
Para ONTSI [7] <<En el desarrollo de la nube en términos de importancia del espectro de servicios ofertados y sobre todo de la calidad, entendida como seguridad y funcionalidad, no solo están desplazando a los gestores de contenidos y aplicativos habituales, sino que están generando una competitividad muy pocas veces vista con anterioridad en ningún otro sector.>>

La solución de Cloud Computing de Software como servicio puede estar orientada a distintos tipos de clientes según su condición:

a) Usuarios particulares (ej: servicios ofimáticos, redes sociales, web 2.0, etc.)

b) Usuarios profesionales (ej: CRM, ERP, etc.)

Los clouds tienen beneficios e inconvenientes, para Areitio [8] son beneficios:

a) Minimiza el gasto de capital y reduce el costo de propiedad (infraestructura como hardware y software) y mantenimiento.

b) Escalabilidad y elasticidad muy alta.

c) No necesita espacio físico para almacenar servidores y bases de datos ya que están en la nube.

d) Independencia de sistemas operativos y virtualmente capacidad de almacenamiento ilimitada.

A su vez, los principales inconvenientes que pueden identificarse son:

(a) La protección de la seguridad y privacidad de datos y programas y

(b) La no portabilidad de una aplicación construida para un servido de nube a otro proveedor de servicios de cloud computing.

Los proveedores más populares de servicios cloud computing son.- Amazon - EC2 (desde 2002) ofrece IaaS, Google - Apps Engine (desde 2008) ofrece PaaS, Microsoft (Windows-Azure), salesfore, etc.

Harris [9] presenta una comparación de los servicios de Cloud Computing, según los modelos de negocio PaaS o IaaS (ver Tabla 2).
Tabla 2 Proveedor-servicio.

\begin{tabular}{lcc}
\hline \multicolumn{1}{c}{ Servicios Cloud } & PaaS & IaaS \\
\hline Amazon aws & $\mathrm{Si}$ & $\mathrm{Si}$ \\
Google App engine & $\mathrm{Si}$ & \\
Windows Azure & $\mathrm{Si}$ & $\mathrm{Si}$ \\
Force.com & $\mathrm{Si}$ & \\
Rackspice & & $\mathrm{Si}$ \\
Gogrid & & $\mathrm{Si}$ \\
\hline
\end{tabular}

\section{APLICACIÓN DE AHP EN SELECCIÓN DE PROVEEDORES DE CLOUD COMPUTING}

En este proceso se selecciona proveedores entre tantos que hay, a su vez se seleccionan criterios para elegir.

\section{Fase 1: Selección de proveedores de Cloud Computing}

Con la finalidad de aplicar AHP en la selección de proveedores de Cloud Computing, se ha tomado en cuenta a proveedores que ofrecen motor de búsqueda propio, y la oferta básica de administración de correos electrónicos, tales como: Google, Microsoft y Yahoo.

\section{Fase 2: Selección de criterios}

Descrita las características de los servicios de Cloud Computing, se ha seleccionado 4 criterios, tales como:

Escalabilidad.- Sencillez con la que se pueden añadir o eliminar recursos.

Accesibilidad.- Los recursos están disponibles en la red y se acceden a través de mecanismos estándar (teléfonos móviles, pc, etc.).

Rendimiento.- El servicio demuestra velocidad y agilidad de respuesta.

Seguridad.- Protege los datos y preserva la privacidad.

Fase 3: Aplicación del Modelo SEPROCCAHP v01

Paso 1 Desarrollo de la estructura jerárquica (modelo jerárquico). Ver Fig. 4. 


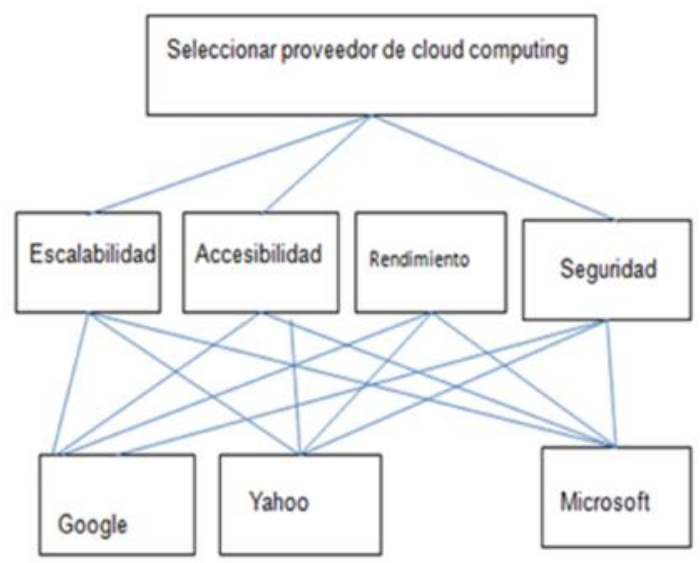

Fig. 4 Modelo jerárquico SEPROCCAHOP v01.

Meta.- Selección de proveedores de Cloud Computing,

Criterios.- Escalabilidad, accesibilidad, rendimiento y seguridad,

Proveedores de Cloud Computing.- Yahoo, Google, Microsoft.

Paso 2 Representación de juicios de valor.Utilizando la escala psicométrica de Saaty se propone el grado de importancia de cada criterio respecto a los otros criterios.

Matriz original

\begin{tabular}{lllll}
\hline Criterio & C1 & C2 & C3 & C4 \\
\hline Flexibilidad (C1) & 1 & $1 / 9$ & $1 / 5$ & $1 / 8$ \\
Escalabilidad (C2) & 9 & 1 & 6 & 2 \\
Seguridad (C3) & 5 & $1 / 6$ & 1 & $1 / 5$ \\
Capacidad (C4) & 8 & $1 / 2$ & 5 & 1 \\
\hline
\end{tabular}

Paso 3 Matrices de juicio de valor.- Matriz original Comparación por parejas acertadas de los tres proveedores para cada criterio.

\begin{tabular}{|c|c|c|c|}
\hline \multicolumn{4}{|c|}{ Con respecto a la flexibilidad } \\
\hline \multirow{2}{*}{ P1 } & $\mathrm{P} 1$ & $\mathrm{P} 2$ & P3 \\
\hline & 1 & 4 & 5 \\
\hline \multicolumn{4}{|l|}{$\mathrm{P} 2$} \\
\hline & $1 / 4$ & 1 & 3 \\
\hline \multicolumn{4}{|l|}{ P3 } \\
\hline & $1 / 5$ & $1 / 3$ & 1 \\
\hline Ponderac. & 0,665 & 0,231 & 0,104 \\
\hline \multicolumn{4}{|c|}{ Con respecto a la escalabilidad } \\
\hline & $\mathrm{P} 1$ & $\mathrm{P} 2$ & P3 \\
\hline \multicolumn{4}{|l|}{ P1 } \\
\hline \multirow{2}{*}{\multicolumn{3}{|c|}{$\mathrm{P} 2$}} & 3 \\
\hline & & & 5 \\
\hline \multicolumn{4}{|l|}{ P3 } \\
\hline & $1 / 3$ & $1 / 5$ & 1 \\
\hline Ponderación & 0,260 & 0,633 & 0,106 \\
\hline \multicolumn{4}{|c|}{ Con respecto a la seguridad $(\mathrm{C} 3)$} \\
\hline & $\mathrm{P} 1$ & $\mathrm{P} 2$ & P3 \\
\hline \multicolumn{4}{|l|}{$\mathrm{P} 1$} \\
\hline & 1 & 3 & 6 \\
\hline P2 & $1 / 3$ & 1 & 3 \\
\hline \multicolumn{4}{|c|}{$1 / 3$} \\
\hline & $1 / 6$ & $1 / 3$ & 1 \\
\hline Ponderación & 0,653 & 0,251 & 0,096 \\
\hline \multicolumn{4}{|c|}{ Con respecto a la capacidad $(\mathrm{C} 4)$} \\
\hline & P1 & $\mathrm{P} 2$ & P3 \\
\hline \multicolumn{4}{|l|}{$\mathrm{P} 1$} \\
\hline \multicolumn{4}{|l|}{ P2 } \\
\hline \multirow[b]{2}{*}{ P3 } & 4 & 1 & $1 / 3$ \\
\hline & 7 & 3 & \\
\hline Ponderación & 0,080 & 0,265 & 0,656 \\
\hline
\end{tabular}

Paso 4 Matriz ajustada.- Los datos de la matriz original sirven para generar la ponderación de los criterios. Las ponderaciones suministran una medida de la importancia relativa de cada criterio.

Tres pasos para calcular la ponderación:

1) Sumar los elementos de cada columna, 
2) Dividir cada valor por la suma de su columna y

3) calcular los promedios de las filas

\begin{tabular}{llllll}
\hline Criterio & C1 & C2 & C3 & C4 & \\
\hline Ponderaciones & 0,040 & 0,512 & 0,113 & 0,335 & 1 \\
\hline
\end{tabular}

Paso 5 Comparaciones de las alternativas de los proveedores.-

\begin{tabular}{llllll}
\hline & C1 & C2 & C3 & C4 & Ponderaciones \\
\hline P1 & $0,04 * 0,665$ & $\ldots$ & $\ldots$ & $\ldots$ & 0,261 \\
P2 & $0,04 * 0,231$ & $\ldots$ & $\ldots$ & $\ldots$ & 0,451 \\
P3 & $0,04 * 0,104$ & $\ldots$ & $\ldots$ & $\ldots$ & 0,289 \\
\hline
\end{tabular}

Paso 6 Razón de consistencia (RC).- Se observa que la razón de consistencia es menor a $10 \%$, por lo que se concluye que es consistente los juicios de valor.

\begin{tabular}{lll}
\hline & Criterio & \% RC \\
\hline Comparación de & Flexibilidad & 8,28 \\
proveedores & & \\
respecto a cada & Escalabilidad & 3,69 \\
criterio & Seguridad & 1,75 \\
& Capacidad & 3,10 \\
Matriz ajustada & & 8,46 \\
& & \\
\hline
\end{tabular}

\section{ANÁLISIS COMPARATIVO DE TRES GRUPOS DE INVESTIGACIÓN DEL CURSO DE LOGÍSTICA EMPRESARIAL}

Se ha simulado el comportamiento de compradores, al observar a tres grupos de investigación del curso de logística empresarial (GP 304 V).

\begin{tabular}{llll}
\hline Factoreslgrupo & A & B & C \\
\hline $\begin{array}{l}\text { Información } \\
\text { sustentada }\end{array}$ & $\mathrm{No}$ & $\mathrm{Si}$ & $\mathrm{Si}$ \\
$\begin{array}{l}\text { Uso de expert } \\
\text { choice }\end{array}$ & $\mathrm{Si}$ & $\mathrm{No}$ & $\mathrm{No}$ \\
$\begin{array}{l}\text { Muestra debate y } \\
\text { discusión }\end{array}$ & $\mathrm{No}$ & $\mathrm{Si}$ & $\mathrm{Si}$ \\
$\begin{array}{l}\text { Proveedor elegido } \\
\text { FIIS UNI 20131 }\end{array}$ & Google & Google & $\begin{array}{l}\text { Micro } \\
\text { soft }\end{array}$ \\
$\begin{array}{l}\text { Consistencia } \\
\text { No }\end{array}$ & Nó & No & Sí \\
\hline
\end{tabular}

\section{CONCLUSIONES}

SEPROCCAHP v01, modelo jerárquico para Seleccionar proveedor de Cloud Computing mediante AHP (PAJ) es aplicable.

La consistencia del resultado depende de los juicios de valor emitidos en la matriz original, así se ha comprobado el comportamiento de los compradores.

\section{AGRADECICIMENTOS}

Agradezco al Dr. Ángel Coca, por brindarme asesoría, al Dr. Mauricio por una interacción inicial en la discusión del tema, a Arthur Huamaní por compartir información sobre Cloud Computing, a Rubén Borja por inducirme en el análisis del tema, a Juan Aldaba por sus aportes, a los alumnos de Logística empresarial 2013-I por aplicar el modelo SEPROCCAHP v01: Rafael Acuña, Carlos Belén; Edgar Beramendi; Carlos Coronel; Deby Guzman y Christian Tiza y a Carlos Zevallos por calcular la consistencia del modelo.

\section{REFERENCIAS}

1. Chopra, S., Meindl, P. “dministración de la cadena de suministros: Estrategia, planeación y operación”. México: Pearson, Prentice Hall, pp. 15, 2008.

2. Saaty, T. "How to make a decision: The Analytic Hierarchy Process. European". Journal of Operational Research 48 (1990) 926 9. North-Holland.

3. INTECO

http://www.cfnavarra.es/observatoriosi/pdf/es tudio_inteco_cloud_computing_en_sector_pu blico/estudio_inteco_cloud_computing_en_se ctor_publico.pdf

4. Rodríguez,I: Pettorutti, J; et.al "Despliegue de un Cloud Privado para entornos de cómputo científico". Universidad de la Plata Argentina. Tomado de http://sedici.unlp.edu.ar/bitstream/handle/109 15/18648/Documento_completo.pdf?sequenc $\underline{\mathrm{e}=1}$ 
5. [5]www.google.com/cloud computing

6. Tiago, S., Rodrigues, J., Costa, J., Proença, M. "E-learning solutions for cloud environments. IEEE latin America Conference On cloud computing and Communications". (2012).

7. ONTSI

http://www.ontsi.red.es/ontsi/es/estudiosinformes/informe-anual-del-sector-ticc-2011edici\%C3\%B3n-2012.

8. Areitio, J., "Protección del Cloud Computing en seguridad y privacidad"

http://dialnet.unirioja.es/servlet/articulo?codi go $=3217380$
9. Harris, Torry. Cloud computing services - a comparison. WP.

10. Grupo Romero. "MICROSOFT UNMSM". 2010

http://noticias.universia.edu.pe/ciencia-nntt/noticia/2010/05/28/694206/unmsm-gruporomero-firman-convenio-microsoft.html

Correspondencia: therehuamani@gmail.com

Recepción de documentos: enero 2014

Aceptación de documentos: mayo 2014 